\title{
PCATool-ADULTO-BRASIL: uma versão reduzida
}

\author{
PCATool-ADULT-BRAZIL: a reduced version \\ PCATOol-ADULTO-BRASIL: una versión reducida
}

\begin{abstract}
Mônica Maria Celestina de Oliveira. Universidade Federal de Ciências da Saúde de Porto Alegre (UFCSPA). Porto Alegre, RS, Brasil. olivmonica@gmail.com (Autora correspondente)

Erno Harzheim. Universidade Federal do Rio Grande do Sul (UFRGS). Porto Alegre, RS, Brasil. ernoharz@terra.com.br

João Riboldi. Universidade Federal do Rio Grande do Sul (UFRGS). Porto Alegre, RS, Brasil. joao.riboldi@ufrgs.br

Bruce Bartholow Duncan. Universidade Federal do Rio Grande do Sul (UFRGS). Porto Alegre, RS, Brasil. bbduncan@ufrgs.br
\end{abstract}

\section{Resumo}

A reorganização do sistema de saúde brasileiro traz a necessidade de avaliação contínua dos serviços ofertados à população. 0 Primary Care Assessment Tool (PCATool-Brasil) versão usuários adultos, validado para o contexto brasileiro, mostrou-se adequado para medir a presença e extensão dos atributos da atenção primária à saúde (APS) nos serviços da saúde. Para otimizar o processo de aplicação e utilização dos resultados em ações estratégicas, é necessária uma versão reduzida deste instrumento. Assim, o objetivo deste artigo é apresentar uma versão reduzida do PCATool-Brasil para usuários adultos e analisar sua adequação. 0 instrumento foi aplicado a 2.404 adultos residentes das áreas adscritas de unidades de APS do município de Porto Alegre no Rio Grande do Sul. Por meio do modelo logístico de dois parâmetros da Teoria de Resposta ao Item (ML-2), foram identificados 23 itens que apresentaram características de discriminação, classificadas de moderada a forte, contemplando os sete atributos da APS. Como medida de consistência, os resultados obtidos com esta versão foram comparados aos resultados da versão completa, revelando escores de APS concordantes. Estes achados indicam que o PCATool-Brasil, versão reduzida para usuários adultos, tem adequada validade e confiabilidade, podendo ser adotado como ferramenta de avaliação rápida de orientação para a APS nos serviços brasileiros, permitindo aos gestores tomada de decisão orientada por evidências para desenvolver ações de melhoria na qualidade dos cuidados ofertados à população.

\section{Abstract}

The reorganization of the Brazilian health system brings the need for on-going evaluation of the services offered to the population. The Primary Care Assessment Tool (PCATool-Brazil) version for adult users, validated for the Brazilian context, adequately measures the presence and extent of attributes of primary health care $(\mathrm{PHC})$ services. A reduced version of this instrument is required to optimize the process of implementation and use of the results in strategic actions. This article aims to present a reduced version of the PCATool-Brazil for adult users and analyze its suitability. The instrument was applied to 2404 adult residents of areas covered by primary health care (PHC) units in Porto Alegre, Rio Grande do Sul state. By the two-parameter logistic model of Item Response Theory (ML-2), 23 items that presented discrimination classified as moderate to strong, contemplating the seven attributes of PHC, were selected. As a measure of consistency, the results obtained with this version were compared with the complete version, revealing consistent PHC scores. These findings indicate that the PCATool-Brazil reduced version for adult users presents adequate validity and reliability, and it can be adopted as a rapid assessment tool to evaluate PHC in Brazilian services, permitting decision making guided by evidence in the development of actions to improve the quality of care offered to the population.

\section{Resumen}

La reorganización del sistema de salud brasileño trae la necesidad de una evaluación continua de los servicios ofrecidos a la población. La Herramienta de Evaluación de Atención Primaria (PCATool-Brasil), versión para usuarios adultos, validada para el contexto brasileño, se mostró adecuada para medir la presencia y el alcance de los atributos de la atención primaria de salud (APS) en los servicios de salud. Para optimizar el proceso de aplicación y utilización de los resultados en acciones estratégicas, se necesita una versión reducida de este instrumento. Así, el objetivo de este artículo es presentar una versión reducida de PCATool-Brasil para usuarios adultos y analizar su adecuación. El instrumento se aplicó a 2.404 adultos residentes en las áreas adscritas a las unidades de APS del municipio de Porto Alegre, en Rio Grande do Sul. A través del modelo logístico de dos parámetros de la Teoría de Respuesta al Ítem (ML-2) se identificaron 23 elementos que mostraron una discriminación clasificada entre moderada y fuerte, contemplando Ios siete atributos de la APS. Como medida de consistencia, los resultados obtenidos con esta versión se compararon con los resultados de la versión completa, revelando puntuaciones de APS concordantes. Estos resultados indican que el PCATool-Brasil, versión reducida para usuarios adultos, tiene validez y fiabilidad adecuadas, y puede ser adoptado como una herramienta de evaluación rápida de orientación para la APS en los servicios brasileños, permitiendo que los gestores puedan tomar decisiones orientados por evidencias para desarrollar acciones destinadas a mejorar la calidad de la atención ofrecida a la población.

Como citar: Oliveira MMC, Harzheim E, Riboldi J, Duncan BB. PCATool-ADULTO-BRASIL: uma versão reduzida. Rev Bras Med Fam Comunidade. 2013; 8(29):256-63. Disponível em: http:// dx.doi.org/10.5712/rbmfc8(29)823

\section{Palavras-chave:}

Atenção Primária à Saúde

Serviços de Saúde

Avaliação de Serviços de Saúde
Keywords:

Primary Health Care Health Services Health Services Evaluation

Palabras clave: Atención Primaria de Salud Servicios de Salud Evaluación de Servicios de Salud

Fonte de financiamento: FAPERGS, CNPq e CAPES. Parecer CEP: 2004367 (Propesq - UFRGS) aprovado em 13/01/2005.

Conflito de interesses: declaram não haver. Recebido em: 17/04/2013 Aprovado em: 23/09/2013 


\section{Introdução}

Em um país de dimensôes continentais como o Brasil, a avaliação sistemática da qualidade dos serviços de saúde disponíveis para a população atendida pelo Sistema Único de Saúde (SUS), abrangendo os diferentes aspectos da atenção, é um grande desafio para os gestores.

Nos últimos anos, a prática de pesquisas para avaliação de serviços de saúde vem ganhando destaque nas agendas públicas e alguns instrumentos, como o Primary Care Assessment Tool (PCATool), se apresentam como uma opção para essas avaliaçóes ${ }^{1-7}$.

A validação deste instrumento, realizada nos EUA, resultou em um conjunto de 92 itens relacionados com confiabilidade e validade suficientes para avaliar aspectos de estrutura e processo da Orientação à Atenção Primária à Saúde (APS) ${ }^{2}$. No Brasil, a versão do PCATool-Infantil aplicada aos cuidadores da população menor de dois anos de idade, usuários dos serviços da rede de atenção básica da regiâo sul de Porto Alegre-RS, foi validada em 2004 e resultou em um instrumento com 45 itens contemplando os atributos essenciais e derivados ${ }^{3,4}$. Depois de uma revisão dessa validação em 2010, uma nova versão mais robusta deste instrumento ficou composta de 55 itens $^{5}$.

Estudo de validação realizado entre 2006-2007 com a versão do PCATool para usuários adultos, também na rede de serviços de Porto Alegre, deu origem a um instrumento composto de 86 itens, que apresentou resultados de confiabilidade e validade semelhantes ao encontrando na validação realizada nos EUA que se revelou uma ferramenta adequada para captar a orientação à APS5 . Em todos os trabalhos de validação do PCATool, a metodologia utilizada foi a Teoria Clássica de Teste (TCT), assim como nas versóes originais da autora do instrumento ${ }^{5-8}$.

Em seguimento aos trabalhos de Starfield et al. ${ }^{2}$ e Harzheim et al. ${ }^{3,4}$, em 2013, foi realizada uma reavaliação do PCATool-ADULTO-BRASIL ${ }^{9}$ utilizando a Teoria da Resposta ao Item (TRI) como metodologia de análise. A partir de um modelo acumulativo unidimensional, os resultados destacaram que há uma contribuição diferente de cada item desse instrumento para o traço latente 'Orientação à APS'. Estes resultados confirmam a adequação do PCATool-Brasil versão usuários adultos para avaliação de serviços de Atenção Primária, evidenciando, no entanto, a baixa contribuição de alguns itens, indicando a possibilidade de redução desse instrumento.

Considerando que a 'Orientação para a APS' é uma informação de extrema relevância para auxiliar gestores da saúde na tomada de decisão para a organização dos serviços, é relevante a estruturação de uma versão reduzida do PCATool-Brasil, para que essa informação possa ser periódica e sistematicamente obtida junto aos usuários dos serviços de APS, configurando um conjunto de painéis longitudinais do grau de orientação à APS dos serviços.

A proposta do presente artigo é apresentar uma versão reduzida do PCATool-Brasil, versão usuários adultos, a partir da reavaliação realizada via Teoria da Resposta ao Item (TRI) por meio da experiência dos usuários dos serviços de APS de Porto Alegre.

\section{Métodos}

\section{Delineamento e fonte de dados}

Um estudo transversal de base populacional realizado entre 2006 e $2007^{1}$, teve como base de avaliação 3.014 sujeitos, adultos maiores de 18 anos de idade, residentes nas áreas geográficas cobertas pela rede pública de Atenção Primária à Saúde (APS) de Porto Alegre e de um serviço privado estruturado sob os princípios da APS. Para este estudo de validação do PCATool, utilizou-se uma subamostra de 2.404 usuários vinculados há pelo menos 12 meses a um dos serviços da rede de Atenção Primária à Saúde do município.

$\mathrm{Na}$ amostra aqui explorada, foram incluídos todos os serviços que funcionaram como primeiro nível do sistema de saúde e foram referidos pelo entrevistado como a sua fonte de cuidado. 


\section{Instrumento de avaliação}

O PCATool-Brasil versão usuários adultos é um instrumento de avaliação da orientação à APS, composto por 86 itens, sendo 3 itens que medem o grau de afiliação do usuário com o serviço de saúde e 85 itens relacionados aos atributos da APS (Primeiro Contato - Utilizaçáo e Acesso, Longitudinalidade, Integralidade - Serviços Disponíveis e Serviços Prestados, Coordenação - Cuidado e Sistema de Informaçôes, Orientação Familiar e Orientação Comunitária).

Estes itens do PCATool-Brasil são descritos de maneira a captar a experiência do usuário com características de processo e estrutura de um serviço de saúde que, quando presentes, qualificam o atendimento prestado à população adscrita ${ }^{10}$.

Em alinhamento ao trabalho desenvolvido na versão completa do PCATool-Brasil ${ }^{5}$, um modelo da Teoria de Resposta ao Item -TRI (Modelo Logístico de 2 parâmetros - ML-2) foi adotado para avaliação das características dos itens para a versão reduzida ${ }^{11}$. Essas características são descritas no contexto deste estudo como discriminação e favorabilidade dos itens para a obtenção do traço latente 'Orientação à APS'.

Como estratégia para reduzir o instrumento, ficou definido que a seleção dos itens seria baseada na discriminação (parâmetro de inclinação do modelo TRI) e na relevância teórica para o escore de orientação à APS. Seguindo essa definição, foi adotado o valor 0,60 como ponto de corte para a estimativa do parâmetro de inclinação, construindo-se assim as seguintes categorias de discriminação:

$$
\begin{gathered}
a_{i}<0,60=\text { baixa } \\
0,60 \leq a_{i} \leq 1,30=\text { moderada } \\
1,31 \leq a_{i} \leq 1,70=\text { alta } \\
a_{i}>1,70=\text { muito alta }
\end{gathered}
$$

Seguindo o referencial teórico adotado por Starfield, todos os investigadores mantiveram, nas versóes adaptadas e validadas em diferentes países, a representatividade dos atributos essenciais e derivados da APS. Dessa forma, a versão reduzida do PCATool-Brasil deveria também apresentar itens desses atributos, garantindo assim uma adequada estimação do escore geral da APS ${ }^{2,4,6-8,12-14}$

Depois da seleção, os itens foram recalibrados na mesma amostra de serviços de APS utilizada na validação da versão do PCATool-ADULTO-BRASIL, a fim de se verificar a reprodutibilidade dos resultados com a estrutura obtida para o instrumento 5 .

Com o objetivo de comparar as versôes do PCATool-Brasil quanto à informação extraída, utilizou-se o mesmo método de obtençâo do escore geral, calculando os escores a partir das médias por atributo. Esse procedimento levou em consideração apenas os itens que foram selecionados por meio do modelo TRI. A consistência interna dessa versáo reduzida foi avaliada por meio do "Alfa de Cronbach" e comparada à versão completa. Valores desse coeficiente acima de 0,70 indicam boa confiabilidade do instrumento. Também se avaliou a concordância entre os escores da versão completa (77 itens) e a versão reduzida (23 itens), por meio do gráfico de Bland-Altman ${ }^{15}$.

Para organização e análise dos dados neste trabalho, foram utilizados os Softwares SPSS 19 e R.15.2 a partir das bibliotecas específicas para abordagem TRI ${ }^{16,17}$.

\section{Aspectos éticos}

O projeto principal intitulado "Avaliaçáo da qualidade do processo de atenção e da sua efetividade sobre a saúde do adulto no Programa Saúde da Família e modelos alternativos no município de Porto Alegre" teve aprovação dos comitês de ética das instituições envolvidas na pesquisa: Secretaria Municipal de Saúde (SMS), Centro de Saúde Escola Murialdo (CSEM), Grupo Hospitalar Conceição (GHC), Grupo de Pesquisa e Pós Graduação - Hospital de Clínicas de Porto Alegre (GPPGHCPA), Caixa de Assistência dos Funcionários do Banco do Brasil - Regional RS (CASSI-RS) e Universidade Federal do Rio Grande do Sul (UFRGS). As entrevistas foram realizadas com os adultos mediante a leitura, aceitação e assinatura do Termo de Consentimento Livre e Esclarecido (TCLE). 


\section{Resultados}

\section{Características da amostra}

As características sociodemográficas da amostra estão apresentadas na Tabela 1.

No que se refere ao grupo de usuários dos cinco serviços de APS, num total de 1.393 sujeitos, observa-se que aproximadamente 63\% referiram uma 'Equipe de Saúde da Família' (ESF) ou uma 'Unidade Básica de Saúde' (UBS), modelos esses existentes em todo o Brasil. $\mathrm{Na}$ amostra geral, observa-se que $24 \%$ dos usuários referiram como referência um 'médico particular' e aproximadamente $13 \%$ citaram o 'ambulatório de um plano de saúde privado'.

Tabela 1. Descrição da amostra de acordo com os serviços avaliados.

\begin{tabular}{|c|c|c|c|c|c|c|c|c|c|}
\hline Serviços & 岕 & $\stackrel{\mathscr{\varrho}}{\mathscr{9}}$ & 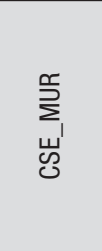 & 옹 & $\begin{array}{l}\bar{W} \\
\text { ऽิ }\end{array}$ & 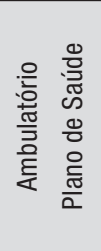 & 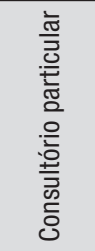 & 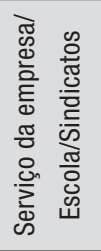 & 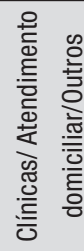 \\
\hline Características sociodemográficas & \multicolumn{9}{|c|}{ Frequências relativas (\%) } \\
\hline Sexo & $\mathrm{n}=578$ & $\mathrm{n}=300$ & $\mathrm{n}=188$ & $\mathrm{n}=134$ & $n=193$ & $\mathrm{n}=301$ & $\mathrm{n}=580$ & $\mathrm{n}=62$ & $\mathrm{n}=68$ \\
\hline Masculino & 34,1 & 37 & 33,5 & 32,1 & 49,7 & 41,9 & 34 & 62,9 & 42,6 \\
\hline Feminino & 65,9 & 63 & 66,5 & 67,9 & 50,3 & 58,1 & 66 & 37,1 & 57,4 \\
\hline Cor (autorreferida) & $\mathrm{n}=576$ & $\mathrm{n}=300$ & $\mathrm{n}=188$ & $\mathrm{n}=134$ & $n=192$ & $n=300$ & $\mathrm{n}=578$ & $\mathrm{n}=62$ & $\mathrm{n}=68$ \\
\hline Não Brancos & 42,9 & 40 & 51,1 & 24,6 & 5,7 & 31,7 & 15,2 & 40,3 & 44,1 \\
\hline Brancos & 57,1 & 60 & 48,9 & $75,4^{*}$ & $94,3^{*}$ & 68,3 & 54,8 & 59,7 & 55,9 \\
\hline Situação conjugal & $\mathrm{n}=578$ & $n=300$ & $\mathrm{n}=187$ & $n=133$ & $n=193$ & $\mathrm{n}=299$ & $\mathrm{n}=578$ & $\mathrm{n}=62$ & $\mathrm{n}=68$ \\
\hline Sem companheiro (a) & 34,9 & 40,7 & 46 & 45,1 & 31,6 & 35,8 & 39,3 & 33,9 & 47,1 \\
\hline Com companheiro (a) & 65,1 & 59,3 & 54 & 54,9 & 68,4 & 64,2 & 60,7 & 66,1 & 52,9 \\
\hline Estrato social & $\mathrm{n}=578$ & $\mathrm{n}=300$ & $\mathrm{n}=188$ & $n=134$ & $n=193$ & $\mathrm{n}=301$ & $\mathrm{n}=580$ & $\mathrm{n}=62$ & $\mathrm{n}=68$ \\
\hline$A B$ & 19,2 & 24,3 & 20,7 & 35,8 & 81,3 & 50,2 & 72,6 & 56,5 & 51,5 \\
\hline CDE & 80,8 & 75,7 & 79,3 & 64,2 & 18,7 & 49,8 & 27,4 & 43,5 & 48,5 \\
\hline Medidas descritivas & \multicolumn{9}{|c|}{ Frequências relativas (\%) } \\
\hline Idade (anos) & $\mathrm{n}=578$ & $n=300$ & $\mathrm{n}=188$ & $n=134$ & $n=193$ & $n=301$ & $\mathrm{n}=580$ & $n=62$ & $\mathrm{n}=68$ \\
\hline Média & 41,89 & 44,21 & 42,8 & 48,8 & $56,4^{*}$ & 44 & 51,9 & 41,1 & 43,4 \\
\hline Mediana & 41 & 43 & 43 & 52 & 55 & 43 & 53,5 & 43 & 42,5 \\
\hline Desvio padrão & 15,1 & 16,8 & 16,4 & 18,3 & 15 & 16,6 & 18,3 & 14,5 & 17,9 \\
\hline Escolaridade (anos ) & $\mathrm{n}=578$ & $\mathrm{n}=297$ & $\mathrm{n}=187$ & $n=134$ & $n=192$ & $\mathrm{n}=299$ & $\mathrm{n}=579$ & $n=62$ & $\mathrm{n}=68$ \\
\hline Média & 6,7 & 7,5 & 7,2 & 8 & 14,8 & 9,8 & 12,2 & 11,5 & 10,06 \\
\hline Mediana & 7 & 8 & 8 & 8 & 15 & 11 & 12 & 11 & 11 \\
\hline Desvio padrão & 3,4 & 3,8 & 3,3 & 3,8 & 3,5 & 3,9 & 4,7 & 4,1 & 3,7 \\
\hline
\end{tabular}

*Diferenças significativas ao nível de 0,05.

Uma análise descritiva dos itens do PCATool apontou para uma contribuição distinta e não nula de todos os itens ao longo da escala de orientação à APS. No entanto, a avaliação via TRI identificou alguns desses itens com baixa discriminação.

Dos itens do PCATool-Brasil organizados de acordo com os atributos da APS, que compóem a versão adulto do PCAToolBrasil, 23 foram selecionados a partir da discriminação e da importância conceitual para a composição da versão reduzida; estes se encontram descritos na Tabela 2 .

A concordância entre os escores da versão completa e a versão reduzida avaliada pelo gráfico de Bland-Altman (Figura 1), apresenta uma distribuição aleatória dos pontos em torno do zero e diferença média de 0,35. 
Tabela 2. Itens do PCATool-ADULTO-BRASIL versão reduzida e estimativas do parâmetro de discriminação do item na versão completa.

\begin{tabular}{|c|c|c|c|}
\hline Atributos da APS & Itens & Descrição do item & Parâmetro de discriminação - ai (EP) \\
\hline Afiliação & $A 1, A 2$ e $A 3$ & $\begin{array}{c}\text { A combinação das } 3 \text { questões referentes ao serviço de } \\
\text { saúde preferencial }{ }^{*}\end{array}$ & $0,874(0,06)$ \\
\hline Primeiro contato [Utilização] & B2 & $\begin{array}{l}\text { Quando tem novo problema vai a esse serviço antes de ir a } \\
\text { outro }\end{array}$ & 0,060 (0,05)\# \\
\hline \multirow{2}{*}{ Primeiro contato [Acesso] } & C4 & Aconselhamento rápido por telefone quando aberto & $1,182(0,064)$ \\
\hline & $\mathrm{C} 11$ & Dificuldade para ter atendimento & $1,072(0,064)$ \\
\hline \multirow{4}{*}{ Longitudinalidade /Atendimento continuado } & D1 & Atendimento pelo mesmo profissional & $0,849(0,057)$ \\
\hline & D6 & Sente-se à vontade & $1,851(0,110)$ \\
\hline & D9 & Sabe quais são os problemas importantes para você & $1,667(0,081)$ \\
\hline & D15 & Mudaria de serviço se fosse fácil & $1,214(0,065)$ \\
\hline \multirow{5}{*}{ Coordenação (Cuidado e Sistemas de informação) } & E6 & Médico sugeriu consulta com especialista & $-0,067(0,059) \#$ \\
\hline & E10 & Informou motivo para consulta & $-0,039(0,061) \#$ \\
\hline & E11 & Resultado da consulta com especialista & $0,727(0,069)$ \\
\hline & E13 & Qualidade da consulta com especialista & $0,927(0,077)$ \\
\hline & F3 & Prontuário disponível & $1,123(0,097)$ \\
\hline \multirow{3}{*}{ Integralidade (Serviços disponíveis) } & G9 & Aconselhamento sobre saúde mental & $0,753(0,053)$ \\
\hline & G17 & Aconselhamento sobre fumo & $0,787(0,053)$ \\
\hline & G20 & Aconselhamento sobre mudanças com o envelhecimento & $1,467(0,075)$ \\
\hline \multirow{4}{*}{ Integralidade (Serviços prestados) } & $\mathrm{H} 1$ & Conselhos sobre a alimentação & $1,743(0,084)$ \\
\hline & H5 & Exercícios apropriados & $1,736(0,084)$ \\
\hline & $\mathrm{H} 7$ & Verifica e discute os medicamentos & $1,908(0,095)$ \\
\hline & $\mathrm{H} 11$ & Prevenção de quedas & $1,361(0,083)$ \\
\hline \multirow{2}{*}{ Enfoque familiar } & 11 & Tratamento e cuidado para você e para a família & $1,910(0,092)$ \\
\hline & 13 & Contato com a família caso solicite & $1,441(0,073)$ \\
\hline Orientação comunitária & J4 & Pesquisas com pacientes & $0,867(0,057)$ \\
\hline
\end{tabular}

*A1- Há um médico ou serviço de saúde onde você geralmente vai se fica doente ou precisa de conselhos sobre a sua saúde? A2 - Há um médico ou serviço de saúde que o/a conhece melhor como pessoa? A3- Há um médico ou serviço de saúde que é mais responsável por seu atendimento de saúde? \# Itens incluídos na versão reduzida conceitualmente.

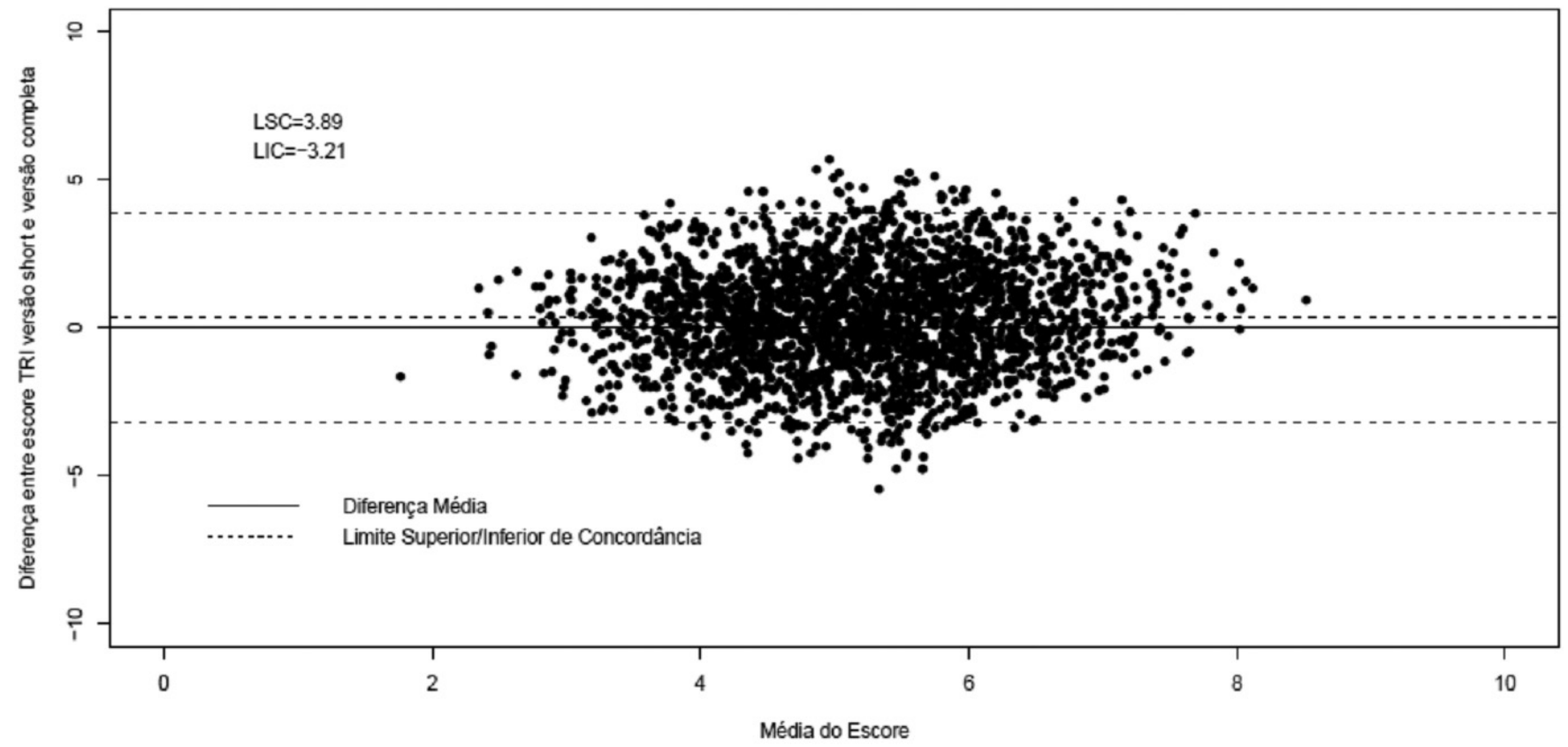

Figura 1. Bland-Altman - Escore TRI versão reduzida vs versão completa. 
A medida de consistência interna dessa versão reduzida, medida pelo $\alpha$ de Cronbach, foi aproximadamente 0,82 . A correlação entre o escore com 77 itens e o escore da versão de 23 itens apresentou uma moderada, mas significativa, associação entre as medidas (coeficiente correlação de Pearson $=0,575, p<0,001$ ). $\mathrm{Na}$ comparação dos escores obtidos via modelo TRI (versão completa e versão reduzida), as diferenças entre os tipos de serviços foram perceptíveis. Os escores para os diferentes serviços e suas respectivas medidas descritivas estão apresentados na Tabela 3.

Tabela 3. Comparação dos escores - versão reduzida por serviços avaliados.

\begin{tabular}{|c|c|c|c|c|c|c|c|c|c|}
\hline Serviços & ESF & UBS & CSE_MUR & $\mathrm{GHC}$ & CASSI & $\begin{array}{l}\text { Ambulatório } \\
\text { Plano de } \\
\text { Saúde }\end{array}$ & $\begin{array}{l}\text { Consultório } \\
\text { Particular }\end{array}$ & $\begin{array}{c}\text { Serviço da } \\
\text { Empresa/ } \\
\text { Escola/ } \\
\text { Sindicatos }\end{array}$ & $\begin{array}{c}\text { Clínicas/ } \\
\text { Atendimento } \\
\text { Domiciliar/ } \\
\text { Outros }\end{array}$ \\
\hline \multicolumn{10}{|c|}{ Medidas descritivas } \\
\hline $\begin{array}{l}\text { Escore TRI Completa } \\
\text { (77 itens) }\end{array}$ & $\mathrm{n}=578$ & $n=299$ & $n=188$ & $n=134$ & $n=193$ & $n=301$ & $n=580$ & $n=62$ & $n=68$ \\
\hline Média & 4,17 & $3,79^{\star}$ & $3,97^{\star}$ & 4,96 & 5,50 & $4,54^{*}$ & 5,30 & 5,09 & 4,51 \\
\hline Mediana & 4,29 & 3,82 & 4,13 & 5,23 & 6,00 & 4,70 & 5,79 & 5,47 & 4,92 \\
\hline Desvio Padrão & 1,75 & 1,68 & 1,71 & 1,77 & 2,22 & 1,57 & 1,93 & 1,75 & 1,98 \\
\hline $\begin{array}{l}\text { Escore TRI Reduzida } \\
\text { (23 itens) }\end{array}$ & $\mathrm{n}=578$ & $\mathrm{n}=299$ & $\mathrm{n}=188$ & $\mathrm{n}=134$ & $n=193$ & $\mathrm{n}=301$ & $\mathrm{n}=579$ & $\mathrm{n}=62$ & $\mathrm{n}=68$ \\
\hline Média & 4,10 & 3,79 & 3,90 & 4,96 & 5,73 & 4,46 & 5,23 & 4,78 & 4,19 \\
\hline Mediana & 4,31 & 4,04 & 4,16 & 5,19 & 6,20 & 4,72 & 5,72 & 5,33 & 4,73 \\
\hline Desvio Padrão & 1,71 & 1,68 & 1,70 & 1,72 & 1,81 & 1,56 & 1,94 & 1,83 & 2,02 \\
\hline $\begin{array}{l}\text { Escore Reduzida } \\
\text { (cálculo por atributos) }\end{array}$ & $\mathrm{n}=578$ & $\mathrm{n}=300$ & $\mathrm{n}=188$ & $n=134$ & $n=193$ & $n=301$ & $\mathrm{n}=580$ & $n=62$ & $n=68$ \\
\hline Média & 5,80 & 5,07 & 5,47 & 6,98 & 7,45 & 6,06 & 6,54 & 6,46 & 5,91 \\
\hline Mediana & 5,75 & 5,02 & 5,41 & 7,07 & 7,65 & 6,06 & 6,64 & 6,68 & 6,05 \\
\hline Desvio Padrão & 1,73 & 1,75 & 1,71 & 1,57 & 1,48 & 1,55 & 1,56 & 1,85 & 1,85 \\
\hline
\end{tabular}

*ANOVA (Análise de Variância) - Diferenças significativas ao nível de 0,05; Serviço de Referência na Comparação - UBS.

Partindo-se da análise das médias dos escores, todos os serviços são classificados como serviços de 'fraca orientação à APS'. Para as ESF e UBS, serviços existentes em todo o país, há uma discreta aproximação das médias, com uma pequena superioridade para a ESF.

Considerando o escore calculado a partir da média por atributos da APS presentes na versão reduzida, percebe-se um considerável aumento nos escores para todos os serviços, com dois destes atingindo médias superiores a 6,6, o que permitiria a classificação como serviços de forte orientação à APS (Tabela 3).

\section{Discussão}

A versão completa do PCATool-ADULTO-BRASIL apresenta itens que são representativos da estrutura e dos processos que norteiam os serviços de APS. No entanto, foi evidenciado no processo de validação via TRI que alguns destes itens contribuíam de forma pouco expressiva para a obtenção do traço latente 'Orientação à APS'. Em funçấo dessa fragilidade considerou-se importante extrair do conjunto de 77 itens aqueles que mais contribuíssem para a obtenção do escore e que tornassem essa ferramenta mais informativa, acessível e útil aos diferentes níveis da gestáo da saúde.

A identificação dos itens que carregam maior quantidade de informação descritiva, sendo, desta forma, aqueles que mais discriminam os serviços quanto ao traço latente, foi embasada diretamente no parâmetro de inclinação do item, permitindo 
aos autores definir que itens com valor deste parâmetro > 0,60 contribuíam, ao menos moderadamente, para o construto 'Orientação para APS'. Esse critério já foi adotado por Castro e Guewehr em seus estudos, também na área da saúde ${ }^{18,19}$.

Avaliando a correlaçáo entre os escores da versão reduzida e completa, foi observado que os escores estáo correlacionados de forma positiva, o que indica que, a partir dessa versão reduzida do instrumento, pode-se seguramente avaliar os serviços de APS.

Assim como foi observado nos estudos de validação do PCATool-Brasil versão infantil ${ }^{3,4}$ e adultos ${ }^{5}$, os itens dos atributos Longitudinalidade e Integralidade-Serviços Prestados são os que apresentam maior contribuição para a estimação do escore. Nesta versão reduzida, também se pode destacar a importância do atributo Orientação Familiar, que apresenta uma elevada contribuição do item, representando a inclusão da família na tomada de decisão quando o paciente julgar importante ${ }^{3,5}$.

Esse achado reforça a capacidade da versão reduzida para captar a contribuição dos itens de maior relevância na descrição de serviços quanto a sua orientação à APS e possibilitar açóes específicas para melhoraria dos serviços ofertados, o que permite sugerir que a versão reduzida seja adotada para avaliaçôes rápidas e sistemáticas dos serviços de APS.

\section{Conclusão}

Alinhada às limitaçóes apontadas na versão completa do PCATool, como a distribuição de itens de diferentes atributos da APS, com baixa contribuição para a estimação do traço latente (orientação à APS) e a consequente interferência nos valores estimados para essa orientação, há uma enorme necessidade de avaliações rápidas e sistemáticas dos serviços de APS no Brasil, para que ajustes e/ou melhorias possam ser implementados, minimizando o impacto dessas fragilidades da APS em outros níveis do sistema.

A versão reduzida do PCATool-ADULTO-BRASIL, aqui proposta, ficou composta por vinte e três itens, contemplando os atributos essenciais e derivados da APS. São eles: Afiliação, Utilização, Acesso - Primeiro Contato, Longitudinalidade, Coordenação do Cuidado e Sistema de Informaçôes, Integralidade - Serviços Disponíveis e Prestados, Enfoque Familiar e Orientaçáo Comunitária. A avaliação de reprodutibilidade da escala na versão reduzida revelou que o instrumento pode seguramente ser utilizado para avaliar os serviços de APS.

\section{Referências}

1. Harzheim E, Duncan BB, Stein AT, Cunha CR, Goncalves MR, Trindade TG, et al. Quality and effectiveness of different approaches to primary care delivery in Brazil. BMC Health Serv Res. 2006 Dec 5; 6: 156. PMid:17147819 PMCid:PMC1790713. http://dx.doi.org/10.1186/1472-6963-6-156

2. Shi L, Starfield B, Xu J. Validating the Adult Primary Care Assessment Tool. J Family Pratice. 2001; 50(2): 161-75.

3. Harzheim E. Evaluación de la atención a la salud infantil del Programa Saúde da Família en la región sur de Porto Alegre, Brasil. [Tese]. Universidad de Alicante; 2004

4. Harzheim E, Starfield B, Rajmil L, Álvarez-Dardet C, Stein TA. Consistência interna e confiabilidade da versão em português do Instrumento de Avaliação da Atenção Primária (PCATool-Brasil) para serviços de saúde infantil. Cad. Saúde Pública. 2006; 22(8): 1649-59. PMid:16832536. http:// dx.doi.org/10.1590/S0102-311X2006000800013

5. Brasil. Ministério da Saúde. Manual do instrumento de avaliação da atenção primária à saúde [Internet]. Brasília: Ministério da Saúde; 2010. Disponível em: http://189.28.128.100/dab/docs/publicacoes/geral/manual_instrumento_avaliacao.pdf

6. Starfield B, Shi L. PCAT Manual. Johns Hopkins University; 2001.

7. Pasarín MI, Berra S, Rajmil L, Solans M, Borrell C, Starfield B. A Tool to Evaluate Primary Health Care From the Population Perspective. Aten Primaria. 2007; 39(8): 395-403. PMid:17692225.

8. Lee JH, Choi Y-J, Sung NJ, Kim SY, Chung SH, Kim J, et al. Development of the Korean primary care assessment tool-measuring user experience: tests of data quality and measurement performance. Int J Qual Health Care. 2009 Apr 1; 21(2): 103-11. PMid:19286829. http://dx.doi.org/10.1093/ intqhc/mzp007

9. Oliveira MMC. Teoria da resposta ao item: aplicação na avaliação de orientação para atenção primária à saúde [Tese]. Universidade Federal do Rio Grande do Sul; 2013.

10. Starfield B. Atenção Primária: equilíbrio entre necessidades de saúde, serviços e tecnologia. Brasília: UNESCO; Ministério da Saúde; 2004. PMCid:PMC1732766. 
11. Andrade DF, Tavares HR, Valle RC. Teoria da Resposta ao Item: Conceitos e Aplicações [Internet]. SINAPE; 2000. Disponível em: http://www. avaliaeducacional.com.br/referencias/arquivos/LivroTRI\%20-\%20Dalton.pdf

12. Macinko J, Almeida C, De Sá PK. A rapid assessment methodology for the evaluation of primary care organization and performance in Brazil. Health Policy Plan. 2007; 22(3): 167-77. PMid:17400576. http://dx.doi.org/10.1093/heapol/czm008

13. Jeon K-Y. Cross-cultural adaptation of the US consumer form of the short Primary Care Assessment Tool (PCAT): the Korean consumer form of the short PCAT (KC PCAT) and the Korean standard form of the short PCAT (KS PCAT). Qual Prim Care. 2011; 19(2): 85-103. PMid:21575331.

14. Berra S, Audisio Y, Mántaras J, Nicora V, Mamondi V, Starfield B. Adaptación cultural y al sistema de salud argentino del conjunto de instrumentos para la evaluación de la atención primaria en salud. Rev Argent Salud Publica. 2011; 2(8): 6-14.

15. Hirakata VN, Camey SA. Análise de concordância entre métodos de bland-altman. Rev HCPA. 2009; 29(3): 261-268

16. International Business Machines - IBM. SPSS Statistics 19 [Internet]. IBM Company; 2010. Disponível em: http://www-01.ibm.com/software/ analytics/spss/

17. R Development Core Team. R: A Language and Enviroment for Statistical Computing [Internet]. Viena: R foundation for Statistical Computing; 2012. Disponível em: http://www.r-project.org/

18. Castro SMJ. Teoria da Resposta ao Item: Aplicação na Avaliação da Intensidade de Sintomas Depressivos [Internet]. [Tese]. Porto Alegre: Universidade Federal do Rio Grande do Sul; 2008 [acesso em 2012 Sept 29]. Disponível em: http://hdl.handle.net/10183/17457

19. Guewehr K. Teoria da Resposta ao Item na avaliação de qualidade de vida de idosos [Internet]. [Dissertação]. Porto Alegre: Universidade Federal do Rio Grande do Sul; 2007 [acesso em 2012 Sept 28]. Disponível em: http://hdl.handle.net/10183/12638 